\title{
Prevalência e fatores associados ao transtorno mental comum em assentados rurais*
}

\author{
Andrécia Cósmem da Silva ${ }^{1}$ \\ Lorena Silva Vargas ${ }^{2,3}$ \\ Rayrane Clarah Chaveiro Moraes ${ }^{4}$ \\ Roselma Lucchese ${ }^{5}$ \\ Rafael Alves Guimarães ${ }^{6}$ \\ Ivania Vera ${ }^{5}$
}

Objetivo: estimar prevalência e fatores associados ao transtorno mental comum em moradores de um assentamento rural. Métodos: estudo transversal realizado em 162 residentes de assentamento localizado na região Centro-Oeste do Brasil. Foi aplicado questionário semiestruturado, a probabilidade de transtorno mental comum obtida através do Self-Reporting Questionnaire (SRQ-20) e a funcionalidade familiar foi avaliada pelo instrumento APGAR de Família. Os dados foram analisados no programa STATA. Resultados: a prevalência de probabilidade de transtorno mental comum foi estimada com intervalo de confiança de 95\%. Foi utilizada a análise de regressão de Poisson com variância robusta para avaliar a associação entre transtorno mental comum e potenciais preditores. A prevalência de possibilidade de transtorno mental foi de $24,1 \%$. As variáveis "sexo feminino", "exposição à violência" e "uso de hipnóticos/sedativos" foram associadas. Conclusão: verificou-se que tais preditores devem ser considerados na atenção à saúde dessa população, no que tange às práticas de prevenção e promoção da saúde, além de refletir a necessidade de articulação de políticas públicas que aproximem a população rural e o setor saúde, independentemente de barreiras territoriais.

Descritores: Prevalência; População Rural; Transtornos Mentais; Atenção Primária à Saúde.

\footnotetext{
* Apoio financeiro da Fundação de Amparo à Pesquisa do Estado de Goiás (FAPEG), Brasil, processo n 201410267000573.

1 Universidade Estadual de Goiás, Ipameri, GO, Brasil.

2 Universidade Federal de Uberlândia, Uberlândia, MG, Brasil.

${ }^{3}$ Prefeitura Municipal de Catalão, Catalão, GO, Brasil.

${ }^{4}$ China Molybdenum, Niobras, Ouvidor, GO, Brasil.

${ }^{5}$ Universidade Federal de Goiás, Catalão, GO, Brasil.

${ }^{6}$ Universidade Federal de Goiás, Goiânia, GO, Brasil.
} 


\section{Prevalence and factors associated with common mental disorder in rural settlers}

Objective: to estimate prevalence and factors associated with common mental disorder in rural settlement dwellers. Methods: a cross-sectional study was carried out in 162 settlement residents located in the Center-West region of Brazil. A semi-structured questionnaire was used, the probability of common mental disorder obtained through the Self-Reporting Questionnaire (SRQ-20) and family functionality was assessed by the Family APGAR instrument. The data were analyzed in the STATA program. Results: the prevalence of probability of common mental disorder was estimated with a $95 \%$ confidence interval. Poisson regression analysis with robust variance was used to evaluate the association between common mental disorder and potential predictors. The prevalence of the possibility of mental disorder was $24.1 \%$. The variables "female sex", "exposure to violence" and "use of hypnotics / sedatives" were associated. Conclusion: it was verified that such predictors should be considered in the health care of this population, regarding health prevention and promotion practices, in addition to reflecting the need for articulation of public policies that approximate the rural population and the health sector, regardless of territorial barriers.

Descriptors: Prevalence; Rural Population; Mental Disorders; Primary Health Care.

\section{Prevalencia y factores asociados al trastorno mental común en asentados rurales}

Objetivo: estimar prevalencia y factores asociados al trastorno mental común en moradores de un asentamiento rural. Método: estudio transversal realizado en 162 residentes de asentamiento localizado en la región Centro-Oeste de Brasil. Se aplicó un cuestionario semiestructurado, la probabilidad de trastorno mental común obtenida a través del Self-Reporting Questionnaire (SRQ-20) y la funcionalidad familiar fue evaluada por el instrumento APGAR de Familia. Los datos se analizaron en el programa STATA. Resultados: la prevalencia de la probabilidad de trastorno mental común fue estimada con un intervalo de confianza del 95\%. Se utilizó el análisis de regresión de Poisson con varianza robusta para evaluar la asociación entre el trastorno mental común y los potenciales predictores. La prevalencia de trastorno mental fue de $24,1 \%$. Las variables "sexo femenino", "exposición a la violencia" y "uso de hipnóticos / sedantes" se asociaron. Conclusión: se verificó que tales predictores deben ser considerados en la atención a la salud de esa población, en lo que se refiere a las prácticas de prevención y promoción de la salud, además de reflejar la necesidad de articulación de políticas públicas que acerquen a la población rural y al sector salud, independientemente de las barreras territoriales.

Descriptores: Prevalencia; Población Rural; Trastornos Mentales; Atención Primaria de Salud. 


\section{Introdução}

Os problemas de ordem psiquiátrica são responsáveis por uma carga significativa de doenças em todo o mundo, correspondendo a um terço do total de morbidades em nível global(1). Os transtornos mentais são patologias manifestadas por um conjunto de sintomas que afetam o indivíduo em suas funções cognitivas, físicas, emocionais e comportamentais, com influência negativa em suas atividades cotidianas, como o trabalho e as relações sociais(2).

Múltiplos fatores têm sido associados à presença de transtorno mental comum (TMC), como idade adulta, sexo feminino, estado civil divorciado ou solteiro, baixa renda familiar, escolaridade, exposição a situações de violência e conflitos familiares ${ }^{(3-5)}$. Além disso, alguns diagnósticos prévios, como a presença de asma, artrite, ataque cardíaco, diabetes, epilepsia, osteoporose e tuberculose são relacionadas à presença de TMC, sobretudo a depressão(6), constituindo quadros de comorbidades.

Entre esses transtornos, reconhece-se o grupo de transtornos mentais comuns (TMC) ou não psicóticos, que engloba o transtorno obsessivo-compulsivo, de ansiedade, depressivo, do pânico, fobias e somatoformes, os quais apresentam elevada prevalência em todo o mundo ${ }^{(7)}$. No âmbito do TMC, pesquisas demonstram elevadas prevalências de depressão maior e ansiedade, com prevalências que oscilam nas diferentes regiões do mundo, a citar,respectivamente, seus valores máximos, 14,5 e 15,5\% na Ásia; 73,6 e 81,8\% na Europa; 49,8 e $69,7 \%$ na América Latina; e 47,0 e $43,7 \%$ no Norte da África ${ }^{(1)}$.

No Brasil, dados sobre a epidemiologia dos TMC em populações rurais são escassos ${ }^{(3-5,8)}$. Em estudo conduzido em 482 assentados rurais de Pernambuco, a prevalência encontrada foi $36,0 \%{ }^{(3)}$. Investigação realizada em área rural do Rio Grande do Norte, estimou uma prevalência de $43,6 \%$ em uma amostra de 61 mulheres $^{(4)}$. Em outro estudo, o percentual de TMC em 163 mulheres residentes da zona rural foi $56,2 \%{ }^{(8)}$. Neste contexto, os residentes de assentamentos rurais constituem uma população com elevada vulnerabilidade psicossocial, pois vivenciam fatores de risco como, precárias condições de vida, baixo poder socioeconômico, transporte precário, escassez de recursos naturais e públicos, pouco apoio social, ausência de serviços de saúde e uso e abuso de álcool, uma vez que estes interferem na qualidade de vida, pois produzem estresse podendo estar relacionados à condições de saúde e favorecendo o desenvolvimento de $\operatorname{TMC}^{(4-5)}$
O fato de existirem relativa escassez de investigação referente à saúde mental em residentes de assentamento rural no país, justifica-se a necessidade de novos estudos que forneçam dados pertinentes para a implementação de estratégias de prevenção a nível primário e secundário, bem como promoção da saúde dos assentados rurais, a fim de auxiliar no planejamento de intervenções e práticas saudáveis voltadas para o bem-estar mental nestas comunidades ${ }^{(4-5,9)}$. Desta forma, o objetivo deste estudo foi estimar a prevalência e fatores associados ao transtorno mental comum em indivíduos de um assentamento rural.

\section{Métodos}

Estudo de corte transversal, de base populacional conduzido em residentes de um assentamento rural localizado na região sudeste do Estado de Goiás, Região Centro-oeste do Brasil, realizado entre setembro e novembro de 2014. O assentamento foi criado oficialmente em 2005, sendo composto por 84 famílias e possui uma população total de 250 indivíduos entre menores e maiores de 18 anos. Os residentes vivenciam precárias condições de vida, como: ausência de água tratada, esgoto, coleta regular de lixo e unidade de saúde local.

Foram considerados os seguintes critérios de inclusão: possuir idade igual ou superior a 18 anos e, residir no assentamento há, no mínimo, seis meses. Indivíduos com diagnóstico médico ou autorrelato de transtorno mental grave e persistente (transtornos psicóticos ou transtorno afetivo bipolar), assim como aqueles que não se encontravam na residência em até três visitas dos pesquisadores de campo foram excluídos do estudo.

Durante o período da investigação, todos os assentados potencialmente elegíveis foram convidados a participar do estudo. O teste piloto foi realizado com quatro indivíduos sorteados aleatoriamente durante uma reunião dos assentados na sede do assentamento, e estes resultados não compuseram o banco de dados do estudo. Os assentados foram abordados em suas residências nos períodos matutino ou vespertino e, orientados sobre objetivos, métodos, benefícios, potenciais riscos da sua participação. Aqueles que concordaram em participar assinaram o Termo de Consentimento Livre e Esclarecido (TCLE) e foram entrevistados, em local privativo.

Os dados foram obtidos por meio de entrevistas, face a face, utilizando um instrumento estruturado sobre as características sociodemográficas, exposição à situação de violência (roubo, brigas, assalto, violência 
sexual, entre outros), uso de substâncias psicoativas, instrumentos de avaliação de funcionalidade familiar e rastreamento de TMC. A média de duração das entrevistas foi de aproximadamente 20 minutos cada.

Para a identificação de possibilidade de TMC, aplicou-se o Self-Reporting Questionnaire (SRQ-20), validado no Brasil e amplamente utilizado em estudos epidemiológicos. Esse instrumento é composto por 20 questões relacionadas às condições de saúde mental não psicótica nos últimos 30 dias. As respostas são do tipo "sim" ou "não", em que cada questão afirmativa pontua com o valor de um. A soma final varia de zero a 20 , em que o escore zero indica nenhuma probabilidade para TMC e o 20, extrema probabilidade para TMC. Escore $\geq$ a sete sugere a presença de $\mathrm{TMC}^{(10)}$.

A funcionalidade familiar foi avaliada pelo instrumento APGAR de família, recomendado pela Organização Mundial da Saúde e pelo Ministério da Saúde. Esse avalia a funcionalidade familiar por meio de cinco itens: Adaptation (Adaptação), Partnership (Companheirismo), Growth (Desenvolvimento), Affection (Afetividade) e Resolve (Capacidade resolutiva). Trata-se de instrumento breve, de custeio baixo e fácil aplicação, e que assessora na identificação precoce da disfuncionalidade familiar. Para cada um dos cinco itens, são atribuídos escores, que variam de zero a 10 pontos, a saber: zero a 4 para elevada disfuncionalidade familiar (EDF); 5 e 6 para moderada disfuncionalidade familiar (MDF); e 7 a 10 para boa funcionalidade familiar (BFF) ${ }^{(11-12)}$.

A variável dependente deste estudo foi a probabilidade de TMC, definido com um escore $\geq 7$ no SRQ-20. Foram avaliadas as seguintes variáveis independentes: idade (anos), sexo, escolaridade, estado civil e possuir filhos. Quanto à religião, foram analisadas três categorias: não possuir religião, religião evangélica e religião católica. Foram também observados: exposição à violência (sofreu ferimentos não fatais resultantes de autoagressões, de agressões interpessoais ou de violência coletiva) $)^{(13)}$; prática regular de atividade física, compreendida em pelo menos 150 minutos de atividade física moderada por semana, ou 75 minutos de atividade física vigorosa, de frequência semanal não determinada, e em sessões com duração de pelo menos 10 minutos $^{(14)}$; uso de hipnóticos ou sedativos prescritos por profissionais de saúde; consumo de álcool na vida; e consumo de tabaco nos últimos 30 dias. Para a disfuncionalidade familiar, consideraram-se os escores $\geq 7$ no instrumento APGAR de família.

Os dados foram analisados no programa STATA, versão 12.0. A prevalência de probabilidade de TMC foi estimada com intervalo de confiança de 95\% (IC95\%). Análise de regressão de Poisson com variância robusta foi utilizada para avaliar a associação entre potenciais preditores e TMC. Para verificar as diferenças entre as proporções foi utilizado o teste qui quadrado. Além disso, foi realizado o teste de correlação de Pearson ( $r$ ) para análise da relação entre o SRQ-20 e o APGAR de família. Foram considerados significantes estatisticamente valores de $p<0,05$.

Esta pesquisa foi aprovada pelo Comitê de Ética em Pesquisa da Universidade Federal de Goiás (protocolo n. 162/2012, CAAE: 33249014.4.0000.5083) e respeitou os princípios éticos de pesquisas envolvendo seres humanos regidos pela Resolução 466/2012.

\section{Resultados}

Do total de residentes do assentamento com idade igual ou superior a 18 anos $(n=200)$, sete recusaram em participar, 10 indivíduos apresentavam diagnóstico médico ou relataram transtorno mental grave e persistente, e 21 não se encontravam na residência em até três visitas. Assim, participaram do estudo 162 assentados, cuja média de idade foi de 41,3 anos. As idades mais prevalentes dos participantes da pesquisa foram $>44$ anos $(47,6 \%),<31$ anos $(27,4 \%)$ e $31-44$ anos $(25 \%)$ respectivamente. Do total de participantes, $54,3 \%$ eram do sexo masculino, escolaridade, $52,9 \%$ possuía menos de oito anos de estudo, 69,2\% eram casados e $47,1 \%$ relataram religião católica.

Tabela 1 - Prevalência e fatores associados ao transtorno mental comum em assentados rurais. Ipameri, GO, Brasil, 2014

\begin{tabular}{|c|c|c|c|c|c|c|}
\hline \multirow{2}{*}{ Variáveis } & \multicolumn{2}{|c|}{$\begin{array}{l}\text { Transtorno mental } \\
\text { comum }\end{array}$} & \multirow{2}{*}{$\begin{array}{l}\mathrm{RP}^{+} \text {bruta } \\
(\mathrm{IC} 95 \%)\end{array}$} & \multirow{2}{*}{ Valor de $p$} & \multirow{2}{*}{$\begin{array}{c}\mathrm{RP}^{\dagger} \\
\text { ajustada } \\
\text { (IC95\%) }\end{array}$} & \multirow{2}{*}{ Valor de $p$} \\
\hline & $\begin{array}{c}\text { Positivol } \\
\text { Total }^{*}\end{array}$ & $\%$ & & & & \\
\hline \multicolumn{7}{|l|}{ Sexo } \\
\hline Masculino & $12 / 88$ & 13,6 & 1,00 & & 1,00 & \\
\hline Feminino & $27 / 74$ & 36,5 & $\begin{array}{c}1,30 \\
(1,08-1,33)\end{array}$ & $<0,01$ & $\begin{array}{c}1,12 \\
(1,02-1,24)\end{array}$ & 0,01 \\
\hline
\end{tabular}




\begin{tabular}{|c|c|c|c|c|c|c|}
\hline \multirow{2}{*}{ Variáveis } & \multicolumn{2}{|c|}{$\begin{array}{c}\text { Transtorno mental } \\
\text { comum }\end{array}$} & \multirow{2}{*}{$\begin{array}{l}\mathrm{RP}^{+} \text {bruta } \\
\text { (IC95\%) }\end{array}$} & \multirow{2}{*}{ Valor de $p$} & \multirow{2}{*}{$\begin{array}{c}\mathrm{RP}^{\dagger} \\
\text { ajustada } \\
\text { (IC95\%) }\end{array}$} & \multirow{2}{*}{ Valor de $p$} \\
\hline & $\begin{array}{c}\text { Positivol } \\
\text { Total* }^{*}\end{array}$ & $\%$ & & & & \\
\hline \multicolumn{7}{|l|}{ Escolaridade (anos) } \\
\hline$>8$ & $14 / 77$ & 18,2 & 1,00 & & 1,00 & \\
\hline$\leq 8$ & $25 / 85$ & 29,4 & $\begin{array}{c}1,09 \\
(0,98-1,21)\end{array}$ & 0,09 & $\begin{array}{c}1,07 \\
(0,97-1,18)\end{array}$ & 0,46 \\
\hline
\end{tabular}

Estado civil

$\begin{array}{lcccc}\text { Solteiro } & 10 / 49 & 20,4 & 1,00 \\ & & & 1,04 & \\ \text { Casado } & 29 / 113 & 25,7 & (0,93-1,16) & 0,46\end{array}$

Filhos

Não

Sim

Religião

Não

Evangélica

Católica

Exposição à violência ${ }^{\ddagger}$

Não

Sim

Prática de atividade física regular

Sim

Não

Consumo de hipnóticos ou sedativos $\$$

$\begin{array}{ll}\text { Não } & \text { 29/1 } \\ \text { Sim } & 10 / 17\end{array}$

Consumo de álcool§

\begin{tabular}{|c|c|c|c|}
\hline Não & $33 / 131$ & 25,2 & 1,00 \\
\hline Sim & $6 / 31$ & 19,4 & $\begin{array}{c}0,95 \\
(0,83-1,08\end{array}$ \\
\hline
\end{tabular}

$\begin{array}{cccc}28 / 132 & 21,2 & 1,00 & \\ 11 / 30 & 36,7 & 1,12 & 0,09 \\ & & & \\ & & & \\ 12 / 54 & 22,2 & 1,00 & \\ 27 / 108 & & 1,02 & \\ & 25,0 & (0,91-1,14) & 0,69\end{array}$

$29 / 145$

$10 / 17$

58,8

1,38
$(1,16-1,63)$

$<0,01$

$(1,05-1,45)$

0,01 


\begin{tabular}{|c|c|c|c|c|c|c|}
\hline \multirow{2}{*}{ Variáveis } & \multicolumn{2}{|c|}{$\begin{array}{l}\text { Transtorno mental } \\
\text { comum }\end{array}$} & \multirow{2}{*}{$\begin{array}{l}\mathrm{RP}^{\dagger} \text { bruta } \\
\text { (IC95\%) }\end{array}$} & \multirow{2}{*}{ Valor de $p$} & \multirow{2}{*}{$\begin{array}{c}\mathrm{RP}^{\dagger} \\
\text { ajustada } \\
\text { (IC95\%) }\end{array}$} & \multirow{2}{*}{ Valor de $p$} \\
\hline & $\begin{array}{l}\text { Positivol } \\
\text { Total* }^{*}\end{array}$ & $\%$ & & & & \\
\hline \multicolumn{7}{|l|}{ Consumo de tabaco§ } \\
\hline Não & $29 / 130$ & 22,3 & 1,00 & & & \\
\hline Sim & $10 / 32$ & 31,2 & $\begin{array}{c}1,07 \\
(0,93-1,22)\end{array}$ & 0,30 & & \\
\hline \multicolumn{7}{|c|}{ Disfuncionalidade familiar } \\
\hline Não & $33 / 145$ & 22,8 & 1,00 & & & \\
\hline Sim & $6 / 17$ & 35,3 & $\begin{array}{c}1,10 \\
(0,92-1,31)\end{array}$ & 0,28 & & \\
\hline
\end{tabular}

*Número de respostas válidas; †RP: razão de prevalência; $¥$ No último ano; §Nos últimos 30 dias

A média da pontuação do SRQ-20 encontrada neste estudo foi de 4,7. A prevalência de possibilidade de TMC nos assentados investigados foi de $24,1 \%$.

A Tabela 1 apresenta a prevalência e fatores associados à probabilidade de TMC. Em análise de regressão múltipla, as seguintes variáveis permaneceram associadas ao desfecho: sexo feminino, exposição à violência e uso de hipnóticos e/ou sedativos.

A Figura 1 representa a correlação entre o APGAR de família e os itens do SRQ-20. Os resultados sugerem correlação negativa entre eles. Assim, um aumento na pontuação do APGAR diminuiu em 2,6 vezes a pontuação do SRQ-20.

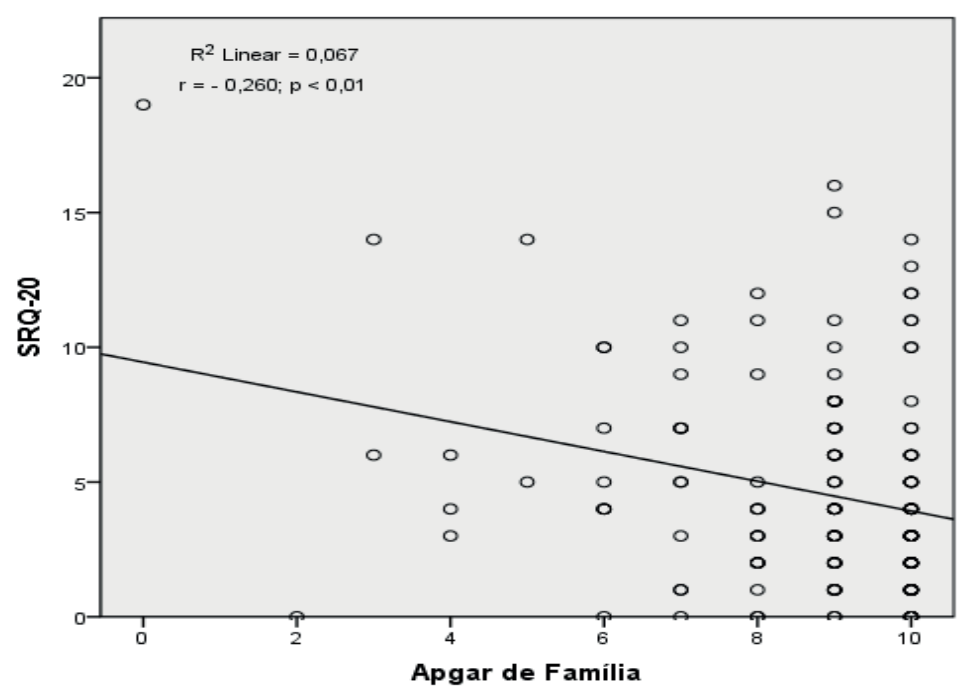

Figura 1 - Correlação entre APGAR de família e Self-Reporting Questionnaire em 162 assentados rurais. Ipameri, GO, Brasil, 2014

\section{Discussão}

O presente estudo analisou a prevalência de probabilidade de TMC e fatores associados em indivíduos que residem em assentamento rural no interior do estado de Goiás, devido a escassez de trabalho referentes à saúde mental da população estudada $^{(4-5)}$. Neste sentido, estudo realizado em zona urbana demonstra prevalência de TMC superior $(31,4 \%)^{(15)}$ que a encontrada na amostra deste estudo $(24,1 \%)$ e menor que o encontrado em outros estudos realizados em assentamentos rurais $(43,6 \%)^{(4-5)}$ e comunidade rural $(36 \%)^{(3)}$. 
A prevalência de probabilidade de TMC varia principalmente por causa do tipo de instrumento diagnóstico, tempo de avaliação dos sintomas e as características da população em estudo. No Brasil, usando os instrumentos General Health Questionnaire (GHQ-12) e Hospital Anxiety and Depression Scale, estimou-se uma prevalência de TMC de $51,9 \%$ no Estado do Rio de Janeiro, 53,3\% em São Paulo, $64,3 \%$ em Fortaleza e $57,7 \%$ em Porto Alegre ${ }^{(15)}$. Sintomas depressivos e transtorno depressivo maior são os mais comuns na população adulta brasileira, sendo que um em cada sete adultos tem sintomas depressivos, e um em cada 12 adultos apresentam transtorno depressivo maior, com prevalências de 14 e $17 \%$, respectivamente ${ }^{(16)}$

Ao ponderar sobre os indivíduos residentes em comunidades rurais de dois países africanos, a prevalência de TMC rastreado pelo SRQ-20 foi de 13,8\% na Etiópia e, de $10,8 \%$ no Quênia, com o uso dos instrumentos Psychosis Screening Questionnaire (PSQ) e Clinical Interview Schedule-Revised (CIS-R) ${ }^{(7,9)}$. A prevalência encontrada neste estudo foi inferior ao estimado em indivíduos de um assentamento rural localizado no Rio Grande do Norte (43,6\%), uma vez observada diferença no tamanho das amostras e em algumas características sociodemográficas das populações, como estado civil e gênero ${ }^{(4-5)}$.

Neste estudo, indivíduos do sexo feminino apresentaram maior prevalência de probabilidade de TMC do que os homens, corroborado por outras investigações ${ }^{(4-5,17-18)}$. Em uma metanálise, que englobou estudos conduzidos em 59 países, mulheres apresentaram taxas ligeiramente maiores de TMC $(19,8 \%)$ do que os homens $(15,2 \%)$, assim como na Polônia e na África, onde as taxas de transtornos depressivos, fóbicos e de humor foram maiores entre as mulheres ${ }^{(17-18)}$. As dificuldades presentes na vida de mulheres de assentamentos rurais as tornam mais vulneráveis ao adoecimento em relação à saúde mental, devido a um cenário de carga de trabalho doméstico e agrícola, violência de gênero, baixa escolaridade e renda, baixo nível de apoio social, relações conjugais e normas culturais ${ }^{(1,4-5)}$.

A presença de transtornos mentais pode ser atribuída à violência, uma vez que indivíduos expostos a eventos traumáticos na comunidade, como acidentes e ameaça com arma, estão mais vulneráveis a agravos, como os transtornos pós-traumáticos ${ }^{(19)}$. Os trabalhadores rurais vinculam os conflitos familiares e violências perpetuadas às situações de vulnerabilidade ao uso de álcool e outras drogas ${ }^{(20)}$.

Estudo aponta prevalência de mulheres do campo que já sofreram agressões ou abuso sexual de $55 \%$, neste sentido casos de violência de gênero contribuem para reprodução da problemática da violência em ambientes rurais, por elementos de ordem socioeconômica e cultural(4-5,20). Apesar de a violência ser um fenômeno complexo, necessitando de maiores aprofundamentos, esse estudo encontrou associação entre TMC e exposição a situações de violência na comunidade.

Do mesmo modo evidenciou-se maior prevalência de probabilidade de TMC em indivíduos que usaram hipnóticos e/ou sedativos nos últimos 30 dias. Essa discussão pode ter dimensões diversas, pois, embora tal associação seja esperada em decorrência do maior uso dessa categoria de fármacos na abordagem terapêutica do TMC, há de se considerar que tais substâncias são responsáveis por desencadear, sobretudo, transtornos de ansiedade e humor ${ }^{(21)}$. Além disso, esses transtornos podem estimular uma busca por substâncias depressoras do sistema nervoso central, em razão do seu efeito amenizador de sintomas desconfortáveis, inerentes às psicopatologias incluídas nos TMC ${ }^{(22)}$. A utilização indiscriminada de psicotrópicos é um problema de saúde a ser enfrentado no contexto rural, visto que a prescrição de medicamentos é o único recurso acessado no cuidado à saúde mental dos indivíduos residentes em zona rural ${ }^{(5,20)}$.

A correlação negativa entre APGAR e SRQ-20 sugere aumento do risco de TMC com o aumento da disfuncionalidade familiar. Desfecho semelhante foi observado em pesquisas realizadas no Brasil e Portugal, com o uso do APGAR de família. No Brasil, houve prevalência de $77,5 \%$, com associação $(p<0,00)$ de sintomas depressivos em idosos com disfunção moderada e/ou elevada ${ }^{(23)}$.Em Portugal, cuidadores de doentes mentais que vivem em áreas rurais e trabalham em um ambiente familiar disfuncional associaram-se a níveis mais elevados de depressão $(p=0,01)$ e estresse $(p=0,00)$. Portanto, indivíduos expostos a ambientes com conflitos familiares apresentam maior risco de TMC, como a depressão(24).

Apesar de não ter sido associada, a variável religião apresenta-se como fator de proteção à saúde mental dos sujeitos em especial àqueles que vivem em situação de maior vulnerabilidade psicossocial como os assentados rurais. A espiritualidade contribui com a melhoria da qualidade de vida dos indivíduos e atribui sentido positivo ao sofrimento(5,25).

As limitações da investigação referem-se à natureza transversal que impossibilita as inferências de relação causa e efeito. Quanto aos instrumentos de rastreamento APGAR de família e o SRQ-20 aplicados, ambos não concluem diagnósticos, sendo necessária uma investigação mais acurada e contextualizada para a 
indicação de um transtorno mental ou disfunção familiar. Os dados foram autorrelatados, ou seja, passíveis de vieses de memória e resposta. No entanto, apontam as possibilidades de forma precoce, de baixo custo $\mathrm{e}$ interdisciplinar para a atenção à saúde em nível primário, com vistas à promoção da saúde.

\section{Conclusão}

Os achados deste estudo indicaram a alta prevalência de TMC em assentados rurais e sua associação com as variáveis sexo feminino, exposição à violência e uso de hipnóticos/sedativos. Esses fatores devem ser apreciados nas práticas preventivas e de promoção da saúde dessa população desassistida no que tange alcance das políticas públicas, sobremaneira quanto às condições de moradia, trabalho, lazer, educação e estilo de vida.

Além disso, o presente trabalho traz à luz a possibilidade de proposição de diretrizes orientadoras de políticas públicas que aproximem a população rural e o setor saúde, independentemente de barreiras territoriais. Faz-se relevante a reflexão acerca das estratégias de articulação intersetoriais ainda incipientes, à fim de realizar ações no âmbito da saúde mental ratificando o cuidado integral e longitudinalidade do atendimento prestado ao indivíduo e coletividade que vivem no campo.

Por fim, os resultados desta investigação explicitam a imprescindibilidade da adoção do SRQ-20 no rastreio da possibilidade de TMC em assentados rurais como forma de sistematização da assistência, assim como contribuem para o enriquecimento de produções na área de Enfermagem, com vistas à instrumentalizar a prática profissional direcionada pela literatura científica, e dessa forma vislumbrar o desenvolvimento de novas pesquisas epidemiológicas focadas nesse grupo populacional.

\section{Referências}

1. Baxter AJ, Patton G, Scott KM, Degenhardt L, Whiteford HA. Global epidemiology of mental disorders: what are we missing? PLoS One. [Internet]. 2013;8(6):e65514. [cited May 9 2016]. Available from: <https://www.ncbi.nlm.nih. gov/pmc/articles/PMC3691161/pdf/pone.0065514.pdf>.

2. American Psychiatric Association (APA). Diagnostic and statistical manual of mental disorders DSM-V. 5th ed. Washington, DC: APA; 2013.

3. Costa AG, Ludermir AB. Common mental disorders and social supportin a rural community in Zona da Mata, Pernambuco State, Brazil. Cad Saúde Pública. [Internet]
2005;21(1):73-9. [cited May 10 2016]. Available from: <http://www.scielo.br/pdf/csp/v21n1/09.pdf>.

4. Costa MGSG, Dimenstein MDB, Leite JF. Condições de vida, gênero e saúde mental entre trabalhadoras rurais assentadas. Estud Psicol. [Internet]. 2014; 19(2):145-54. [cited May 6 2016]. Available from: <http://www.scielo.br/ pdf/epsic/v19n2/07.pdf>.

5. Costa MG, Dimenstein M, Leite J. Estratégias de Cuidado e Suporte em Saúde Mental Entre Mulheres Assentadas. Reva Colombiana Psicol. [Internet]. 2015; 24(1):13-27. [cited May 2 2016]. Available from: <https://revistas.unal.edu.co/index.php/psicologia/article/ view/41968/61911>.

6. Jackson JC, Pandharipande PP, Girard TD, Brummel NE, Thompson JL, Hughes CG, et al. Depression, Posttraumatic Stress Disorder, and Functional Disability in Survivors of Critical Illness: results from the BRAIN ICU (Bringing to light the Risk Factors And Incidence of Neuropsychological dysfunction in ICU survivors) Investigation: A Longitudinal Cohort Study. Lancet Respir Med. [Internet]. 2014;2(5):369-79. [cited Apr 27 2016]. Available from: <https://www.ncbi.nlm.nih.gov/pmc/ articles/PMC4107313/pdf/nihms595830.pdf>.

7. Jenkis R, Njenga F, Okonji M, Kigamwa P, Makheti B, Ayuyo J, et al. Prevalence of Common Mental Disorders in a Rural District of Kenya, and Socio-Demographic Risk Factors. Int J Environ Res Public Health [Internet]. 2012;9(5):1810-9. [cited May 25 2016]. Available from: <http://www.mdpi.com/1660-4601/9/5/1810/htm>.

8. Paffer AT, Ferreira AS, Cabral Júnior CR, Miranda CT. Prevalence of common mental disorders in mothers in the semiarid region of Alagoas and its relationship with nutritional status. Sao Paulo Med J. [Internet]. 2012;130(2):84-91. [cited May 13 2016]. Available from: <http://www.scielo.br/pdf/spmj/v130n2/03.pdf>.

9. Fekadu A, Medhin G, Selamu M, Hailemariam M, Alem A, Giorgis TW, et al. Population level mental distress in rural Ethiopia. BMC Psychiatry. [Internet]. 2014;14:194. [cited 13 Apr 27 2016]. Available from: <https://bmcpsychiatry. biomedcentral.com/articles/10.1186/1471-244X-14-194>. 10. Gonçalves DM, Stein AT, Kapczinski F. Performance of the Self-Reporting Questionnaire as a psychiatric screening questionnaire: a comparative study with Structured Clinical Interview for DSM-IV-TR. Cad Saúde Pública. [Internet]. 2008;24(2):380-90. [cited May 4 2016]. Available from: <http://www.scielo.br/pdf/csp/v24n2/16.pdf>.

11. World Health Organization (WHO). Latino families and youth: a compendium of assessment tools. [Internet] Geneva: WHO; 2012. [cited Apr 18 2016]. Available from: $<$ http://www.rehueong.com.ar/sites/default/files/Binderorpinas $\% 20$ meassurement.pdf>.

12. Brasil. Ministério da Saúde. Secretária de Atenção à Saúde. Departamento de Atenção Básica. Envelhecimento 
e saúde da pessoa idosa. [Internet]. Brasília, DF: Ministério da Saúde; 2007. [cited June 10 2016]. Available from: <http://bvsms.saude.gov.br/bvs/publicacoes/abcad19.pdfs. 13. Dahlberg LL, Krug EG. Violence: a global public health problem. Ciênc Saúde Coletiva. [Internet]. 2007;11(Sup):1163-78. [cited May 2 2016]. Available from: <http://www.scielo.br/pdf/csc/v11s0/a07v11s0.pdf>.

14. World Health Organization (WHO). Global recommendations on physical activity for health. [Internet]. Geneva: WHO; 2010. [cited May 18 2016]. Available from: <http://apps.who.int/iris/bitstre am/10665/44399/1/9789241599979_eng.pdf>.

15. Gonçalves DM, Mari JJ, Bower P, Gask L, Dowrick C, Tófoli LF, et al. Brazilian multicentre study of common mental disorders in primary care: rates and related social and demographic factors. Cad Saúde Pública. [Internet]. 2014;30(3):623-32. [cited Apr 29]. Available from: $<$ http://www.scielo.br/pdf/csp/v30n3/0102-311Xcsp-30-3-0623.pdf>.

16. Silva MT, Galvão TF, Martins SS, Pereira MG. Prevalence of depression morbidity among Brazilian adults: a systematic review and meta-analysis. Rev Bras Psiquiatr. [Internet]. 2014;36(3):262-70. [cited May 7 2016]. Available from: <http://www.scielo.br/pdf/rbp/v36n3/15164446-rbp-2014-36-3-262.pdf>.

17. Steel Z, Marnane C, Iranpour C, Chey T, Jackson J, Patel $\mathrm{V}$, et al. The global prevalence of common mental disorders: a systematic review and meta-analysis 19802013. Int J Epidemiol. [Internet]. In2014;43(2):476-93. [cited May 24 2016]. Available from: <https://www.ncbi. nlm.nih.gov/pmc/articles/PMC3997379/pdf/dyu038.pdf>. 18. Angst J, Paksarian D, Cui L, Merikangas KR, Hengartner MP, Ajdacic-Gross V, et al. The epidemiology of common mental disorders from age 20 to 50 : results from the prospective Zurich cohort Study. Epidemiol Psychiatr Sci. [Internet]. 2015:1-9. [cited Apr 28 2016]. Available from: $<$ https://www.cambridge.org/core/services/aop-cambridgecore/content/view/BD574C83A6E08925E95D51DE2C4381 9B/S204579601500027Xa.pdf/epidemiology_of_common_ mental_disorders_from_age_20_to_50_results_from_the prospective_zurich_cohort_study.pdf>.

19. Ribeiro WS, Andreoli SB, Ferri CP, Prince M, Mari JJ. Exposure to violence and mental health problems in low and middle-income countries: a literature review. Rev Bras Psiquiatr. [Internet]. 2009; 31(Supl II):S49-57. [cited May 18 2016]. Available from: <http://www.scielo.br/pdf/rbp/ v31s2/en_v31s2a03.pdf>.

20. Costa Neto MC. Cuidado Psicossocial em Saúde Mental: Estudo em Assentamentos Rurais do Rio Grande do Norte [dissertação]. Rio Grande do Norte (RN): Universidade Federal do Rio Grande do Norte; 2016. 177p.

21. Blanco C, Rafful C, Wall MM, Jin CJ, Kerridge B, Schwartz RP. The latent structure and predictors of non- medical prescription drug use and prescription drug use disorders: a national study. Drug Alcohol Depend. [Internet]. 2013;133(2):473-9. [cited June 2 2016]. Available from: <https://www.ncbi.nlm.nih.gov/pmc/articles/PMC3818293/ pdf/nihms-517015.pdf>.

22. Swendsen J, Conway KP, Degenhardt L, Glantz M, Jin $\mathrm{R}$, MeriKangas $\mathrm{KR}$, et al. Mental disorders as risk factors for substance use, abuse and dependence: results from the 10- year follow-up of the National Comorbidity Survey. Addiction. [Internet]. 2010;105(6):1117-28. [cited June 2 2016]. Available from: <https://www.ncbi.nlm.nih.gov/pmc/ articles/PMC2910819/pdf/nihms188508.pdf>.

23. Souza RA, Costa GD, Yamashita CH, Amendola F, Gaspar JC, Alvarenga MRM, et al. Family functioning of elderly with depressive symptoms. Rev Esc Enferm USP. [Internet]. 2014;48(3):469-76. [cited May 18 2016]. Available from: <http://www.scielo.br/pdf/reeusp/v48n3/0080-6234reeusp-48-03-469.pdf>.

24. Cabral L, Duarte J, Ferreira M, Santos C. Anxiety, stress and depression in family caregivers of the mentally ill. Aten Primaria. [Internet]. 2014;46(Suppl 5):S176-9. [cited May 23 2016]. Available from: <https://ac.els-cdn. com/S0212656714700873/1-s2.0-S0212656714700873main.pdf?_tid=b39be582-d3e2-49fa-8572-0c7f17ee91f a\&acdnat $=1536584048 \_6758 b 609 c 096 a c a 8 c 7591332$ e9936e1c>.

25. Gonçalves AMS, Santos MA, Pillon SC. Uso de álcool e/ou drogas: avaliação dos aspectos da espiritualidade e religiosos. SMAD, Rev Eletrônica Saúde Mental Álcool Drog. [Internet]. 2014; 10(2): 61-9. [cited May 26 2016]. Available from: <http://pepsic.bvsalud.org/pdf/smad/v10n2/ en_03.pdf>. 